\title{
Quadraparesis following Chiropractic Manipulation: A Case Report
}

\section{Behnoosh Tabloie ${ }^{1}$ and Arun Aggarwal ${ }^{2 *}$}

${ }^{1}$ Rehabilitation Registrar, Balmain Hospital, Balmain, NSW, Australia 2041

${ }^{2}$ Clinical Associate Professor, Department of Rehabilitation Medicine, Royal Prince Alfred Hospital, Camperdown, NSW, Australia 2050

\section{Abstract}

Chiropractic manipulation is a popular form of alternative therapy used in the treatment of acute and chronic pain. Neurological complications are uncommon, occurring in 1 per million cervical manipulations.

We report a case of a 35-year old man who developed a C3/C4 quadraparesis, shortly after chiropractic manipulation to his neck for acute on chronic neck pain. A CT scan of his cervical spine showed a large central disc protrusion at C3/4 causing severe cord compression and underwent urgent C3/4 cervical decompression with fusion. He improved neurologically and after a period of inpatient rehabilitation he has discharged home, 3 weeks after presentation, independent in mobility and self care with minimal residual upper limb weakness, which resolved over the next few months.

Even though neurological complications of spinal manipulation are well recognised, our case demonstrates the dangers of this popular form of alternative therapy that is generally seen to be benign.

Lessons: Chiropractic manipulation is a popular form of alternative therapy used in the treatment of acute and chronic pain and neurological complications are uncommon, occurring in 1 per million cervical manipulations.

Even though neurological complications of spinal manipulation are well recognised, our case demonstrates the dangers of a therapy that is generally seen to be benign.

If the complication is identified as a neurological emergency, excellent functional recovery occurs.

This case highlights the potential pitfalls of this form of treatment and the need for more strict medical control to minimise the risk of complications, especially at the cervical level.

Introduction: Chiropractic spinal manipulation involves a high-velocity thrust to rapidly adjust joints and correct spinal misalignment and relieve pressure on nerves to allow them to function.

Neurological complications of cervical manipulations are considered rare, occurring in 1 per million cervical manipulations [1]. Most complications reported of this therapy are case reports of vertebral artery dissection [2], disc herniation [3] and cervical fracture [4], but there have been reports of Brown-Sequard syndrome due to cord contusion [5], acute paraplegia due to ischaemia [6] and hemiplegia due to a spinal epidural haematoma [7]

Even though neurological complications of spinal manipulation are well recognized [8,9] our case demonstrates the dangers of this popular form of alternative therapy.

Keywords: Quadraparesis; Chiropractic

\section{Case Report}

A 35-year old man had a 2-year history of chronic cervical pain, and had received chiropractic manipulation, with partial and temporary short-term relief of symptoms. He had an acute exacerbation of pain and stiffness and underwent chiropractic manipulation that lasted for about half an hour. During the treatment session, he experienced an electric shock like sensation and then noticed some numbness of the left side of his body. Over the next hour, he developed progressive weakness of his upper and lower limbs with reduced sensation. He had become incontinent of urine and faeces.

Neurological examination revealed a flaccid quadraparesis with MRC grade $0 / 5$ power in all limbs and a sensory level to touch, pain and temperature from C5. Deep tendon reflexes were brisk in the upper and lower limbs. His plantar responses were extensor.

An urgent CT scan of the cervical spine was performed, which showed a large posterior disc protrusion centrally at $\mathrm{C} 3 / 4$ resulting in significant cord compression, with approximately $65 \%$ narrowing of the canal. There was no evidence of vertebral artery dissection Figure 1.

He was commenced on intravenous Dexamethasone and underwent urgent $\mathrm{C} 3 / 4$ cervical decompression with fusion. The following day after surgery, he had some return of power on the right side and two days later, was partially able move the left side of the body. He was placed in a Miami J collar to stabilise his cervical spine and transferred for a period of inpatient rehabilitation.

Following further physical therapy, he was able to walk independently and became independent in all activities of daily living. He had residual distal left upper limb weakness of wrist flexion and extension of MRC grade 4/5 and finger abduction and flexion of 3/5. His left upper limb reflexes remained brisk but his sensory examination returned to normal to touch, pain and temperature.

During his rehabilitation program, he developed neuropathic pain in his left upper limb with sharp, stabbing and knife-like pain with no burning sensation. This responded well to a small dose of a tricyclic

*Corresponding author: Arun Aggarwal, Department of Rehabilitation Medicine, Royal Prince Alfred Hospital, Camperdown, NSW, Australia 2050, Tel: +612 9515 9815; Fax: +612 9817 6633; E-mail: arun.a@sydney.edu.au

Received April 09, 2012; Accepted April 18, 2012; Published April 30, 2012

Citation: Tabloie B, Aggarwal A (2012) Quadraparesis following Chiropractic Manipulation: A Case Report. J Clin Case Rep 2:137. doi:10.4172/2165 7920.1000137

Copyright: @ 2012 Tabloie B, et al. This is an open-access article distributed unde the terms of the Creative Commons Attribution License, which permits unrestricted use, distribution, and reproduction in any medium, provided the original author and source are credited. 
Citation: Tabloie B, Aggarwal A (2012) Quadraparesis following Chiropractic Manipulation: A Case Report. J Clin Case Rep 2:137. doi:10.4172/21657920.1000137
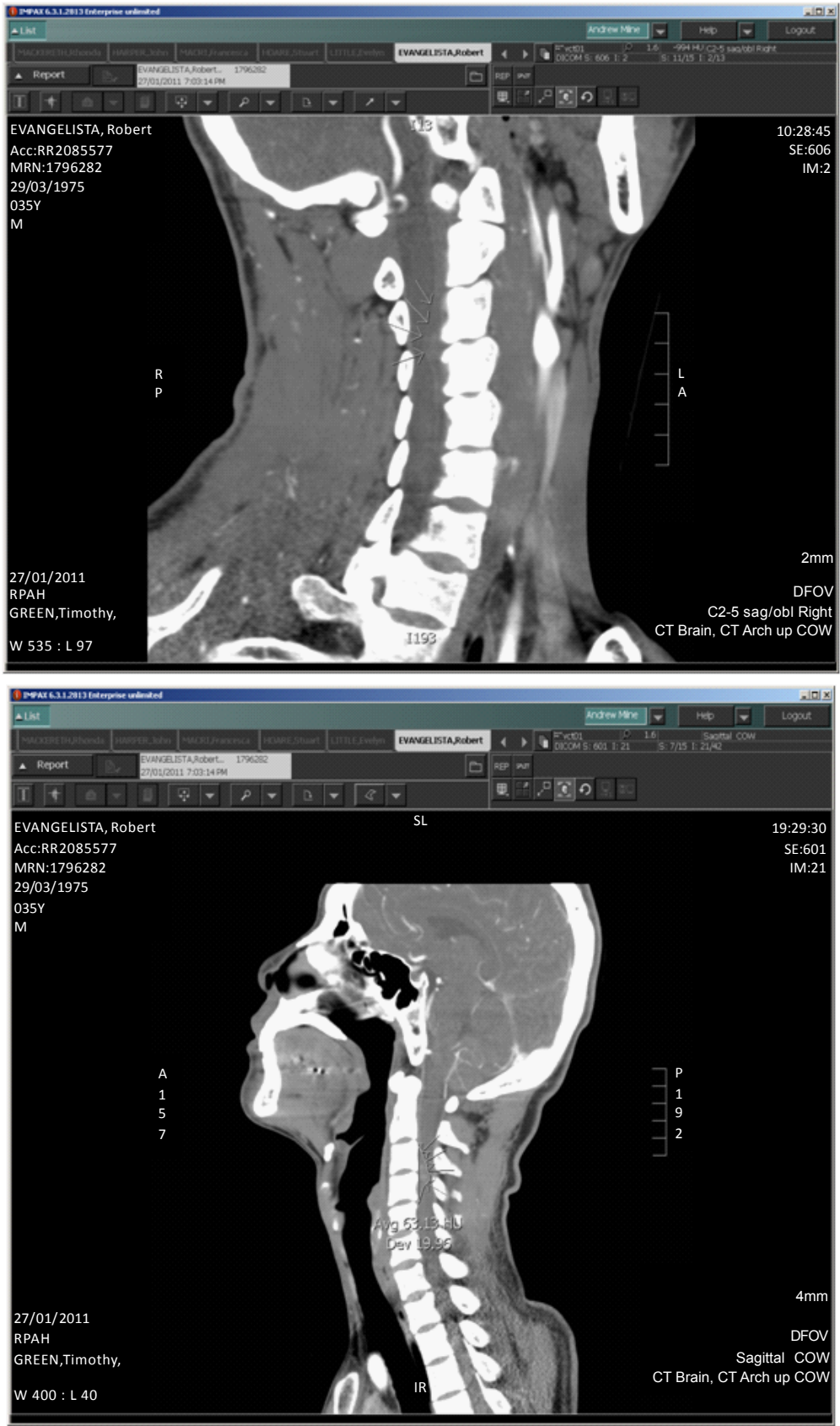

Figure 1: Sagittal CT scan of the cervical spine with arrows showing the large posterior disc protrusion centrally at C3/4 resulting in cord compression.

anti-depressant and an anti-convulsant agent. He was discharged home, 3 weeks after his initial presentation.

He was reviewed by his neurosurgeon after 6 weeks and advised to cease wearing the hard collar. He still had reduced range of movement of his cervical spine with forward flexion $2 \mathrm{~cm}$ from the chest, extension to 20 degrees, lateral flexion to 30 degrees and lateral rotation to 45 degrees. His left upper limb power had normalised with MRC grade $5 / 5$ power of shoulder abduction, wrist flexion and finger flexion. As a result, he was able to return to work as an information technology officer and also return to driving a car.

Over the next 3 months, he continued to recover, obtaining full range of movement of his cervical spine and normal power and sensation in the upper limbs. This only residual neurological sign that persists is that his upper limb reflexes remain brisk.

\section{Discussion}

Chiropractic spinal manipulation is a popular form of alternative therapy used in the treatment of acute and chronic neck pain and headaches. It often involves high-velocity thrusts to rapidly adjust joints and correct spinal misalignment and relieve pressure on nerves. When manipulation is performed, a joint is moved further than is normally possible in an active movement. Passive mobilisation moves a joint through its normal range of motion. There is however no evidence to indicate that neck manipulation by a chiropractor is any more effective for relieving mechanical neck disorders than a number 
of other physical treatment modalities, and it is clear that adverse reactions are more likely to occur following manipulation [8].

Before cervical manipulation is commenced for sudden onset neck pain, it is essential that an attempt be made to rule out a vertebral artery dissection, as manipulation may release an embolus. One of the most devastating injuries reported following a chiropractic adjustment is stroke. In a Canadian survey, dissection of the vertebral arteries was one of the most common causes of stroke in patients less than 45 years of age [10]. It has been estimated that about $20 \%$ of all basilar strokes are due to spinal manipulations [11].

Cervical manipulation is contraindicated in patients with rheumatoid arthritis, acute fractures, dislocations, infection of bone, osseous malignancies, cervical myelopathy, vertebro-basilar insufficiency, aneurysms of a major blood vessel and patients on anticoagulation therapy. It is relatively also contraindicated in cases of joint hyper-mobility and osteoporosis [1].

It is also contraindicated in the acute phase of cervical disc herniation until the neurological deficit has resolved. Dvorak et al. [12] advised that in the acute phase of cervical disc herniation with neurologic deficit, manipulation and mobilisation of the affected segments are contraindicated as there is a high risk of spinal cord compression due to massive prolapse. Inappropriate cervical spine manipulation may force excessive movement and worsen symptoms related to cervical disc herniation or spondylosis, producing such complications as radiculopathy and myelopathy [12].

Neurological complications are uncommon, occurring in one per million cervical manipulations [1]. Most reported complications of this therapy are case reports of vertebral artery dissection [2], disc herniation [3] and cervical fracture [4], but there have been reports of BrownSequard syndrome due to cord contusion [5], acute paraplegia due to ischaemia [6] and hemiplegia due to a spinal epidural haematoma [7].

Even though neurological complications of spinal manipulation are well recognized $[8,9]$, our case demonstrates the dangers of this popular form of alternative therapy that is generally seen to be benign. Fortunately, this complication was identified as a neurological emergency and made an excellent functional recovery. This case highlights the potential pitfalls of this form of treatment and the need for more strict medical control to minimise the risk of complications, especially at the cervical level.

\section{References}

1. Assendelft WJ, Bouter LM, Knipschild PG (1996) Complications of spinal manipulations: a comprehensive review of the literature. J Fam Pract 42: 475480.

2. Chen WL, Chern CH, Wu YL, Lee CH (2006) Vertebral artery dissection and cerebellar infarction following chiropractic manipulation. Emerg Med J 23: e1.

3. Paterson JK (2001) Neurological complications of cervical spine manipulation J R Soc Med 94: 314-315.

4. Ziv I, Rang M, Hoffman HJ (1983) Paraplegia in osteogenesis imperfecta - A case report. J Bone Joint Surg Br 65: 184-185.

5. Lipper MH, Goldstein JH, Do HM (1998) Brown-Sequard syndrome of the cervical spinal cord after chiropractic manipulation. AJNR Am J Neuroradiol 19: $1349-1352$.

6. Lopez-Gonzalez A, Peris-Celda M (2010) Acute paraplegia after chiropraxis Eur Spine J 20: S143-S146.

7. Lidder S, Lang KJ, Masterson S, Blagg S (2010) Acute spinal epidura haematoma causing cord compression after chiropractic neck manipulation: an under-recognised serious hazard? J R Army Med Corps 156: 255-257.

8. Stevinson C, Honan W, Cooke B, Ernst E (2001) Neurological complications of cervical spine manipulation. J R Soc Med 94: 107-110.

9. Stevinson C, Ernst E (2002) Risks associated with spinal manipulation. Am J Med 112: 566-571.

10. Chan MT, Nadareishvili ZG, Norris JW (2000) Diagnostic strategies in young patients with ischemic stroke in Canada. Can J Neurol Sci 27: 120-124.

11. Rothwell DM, Bondy SJ, Williams JI (2001) Chiropractic manipulation and stroke: a population-based case-control Study. Stroke 32: 1054-1060.

12. Dvorák J, Loustalot D, Baumgartner H, Antinnes JA (1993) Frequency of complications of manipulation of the spine. A survey among the members of the Swiss Medical Society of Manual Medicine. Eur Spine J 2: 136-139. 